FERMILAB-TM-2664-DI

October 2017

\title{
Accelerator/Experiment Operations - FY 2017
}

\author{
M. Convery, K. Decker ${ }^{1}$, L. Fields, B.T. Fleming, S. Geer ${ }^{2}$, D. Geesaman, D. Harris, D. Johnson, T. \\ Kobilarcik, M. Messier, D. Newhart, P. E. Reimer, M. Rominsky, J.J. Schmidt, P. Shanahan, G.P. \\ Zeller
}

\section{Introduction}

This Technical Memorandum summarizes the Fermilab accelerator and experiment operations for FY 2017. It is one of a series of annual publications intended to gather information in one place. In this case, the information concerns the FY 2017 NOvA, MINOS+ and MINERvA experiments using the Main Injector Neutrino Beam (NuMI), the MicroBooNE experiment and the activities in the SciBooNE Hall using the Booster Neutrino Beam (BNB), and the SeaQuest experiment, LArIAT experiment and Meson Test Beam activities in the $120 \mathrm{GeV}$ external switchyard beam (SY120).

Each section was prepared by the relevant authors, and was then edited for inclusion in this summary.

\section{Accelerator Operations (M. Convery, D. Johnson, D. Newhart)}

The low-energy and high-energy neutrino beams, the SeaQuest beam, and the test beams in the Meson area were brought into operation after the summer 2016 accelerator shutdown. The Booster Neutrino Beam startup occurred on October $14^{\text {th }}$, and NuMI beam started up on November $11^{\text {th }}$. Beam was delivered to users until the present shutdown which began July $7^{\text {th }}$. Commissioning of Muon Campus beam to the g-2 experiment began on April $7^{\text {th }}$ and beam was delivered to the experiment for detector commissioning from June $1^{\text {st }}$ until the shutdown.

The high-energy neutrino beam was scheduled for beam delivery for 34 weeks. NuMI beam power ramped up quickly to $700 \mathrm{~kW}$ once the new Recycler collimators were commissioned. There were 4823 hours of high-energy operation in FY 2017. Integrated beam to NuMI met expectations for the year.

The Booster Neutrino Beam exceeded base goals. The flux was reduced when NuMI started running at $700 \mathrm{~kW}$, but was restored after the approval of a new Booster shielding assessment. About 5545 hours of beam was delivered to the MicroBooNE experiment.

\footnotetext{
${ }^{1}$ Administrative Support

${ }^{2}$ Editor
} 
Beam delivery to SeaQuest, including commissioning time, was 3177 hours with $8.16 \times 10^{17}$ protons delivered. The slow spill beam had the desired intensity and good extraction efficiency.

The Fermilab Test Beam Facility was scheduled for beam for 26 weeks of the 52-week period. Beam delivery started on November $24^{\text {th }}$ and continued until July $7^{\text {th }}$. A total of $6.12 \times 10^{15}$ protons were delivered. The facility operated for 1780 hours driven by user requests. The failure of one of the MI extraction septa in December caused about a month of downtime and required rescheduling of test beam users.

The LArIAT experiment located in the MCenter beam restarted operation in March. Beam was delivered to the experiment for 1172 hours as requested, with $1.10 \times 10^{9}$ protons delivered.

The main summer 2017 shutdown drivers are completion of the Recycler vacuum TSP to ion pump upgrades, the installation of new Marx modulators for the Linac RF, and further Muon Campus installation work.

The following plots summarize the beam delivery in FY 2017.

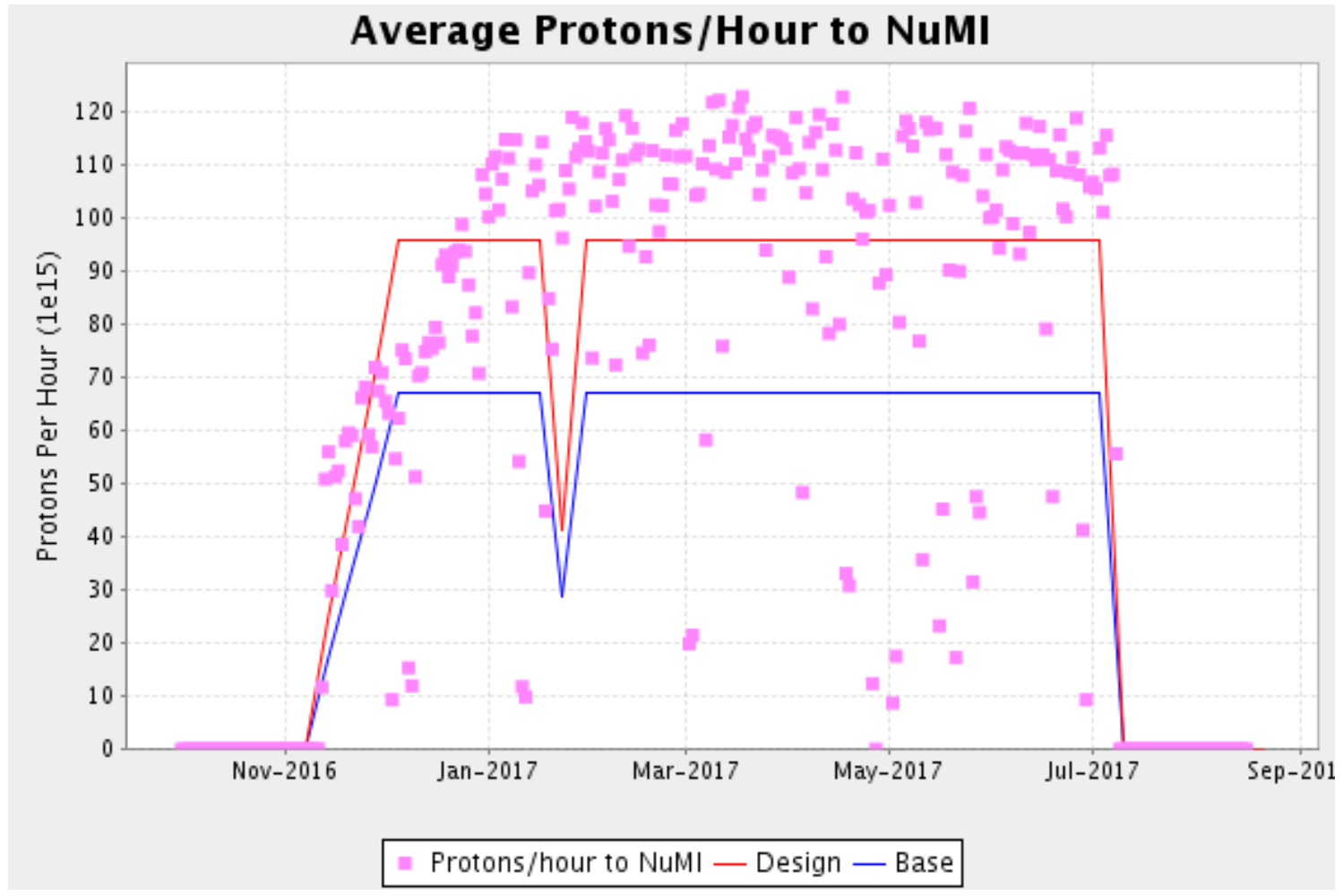



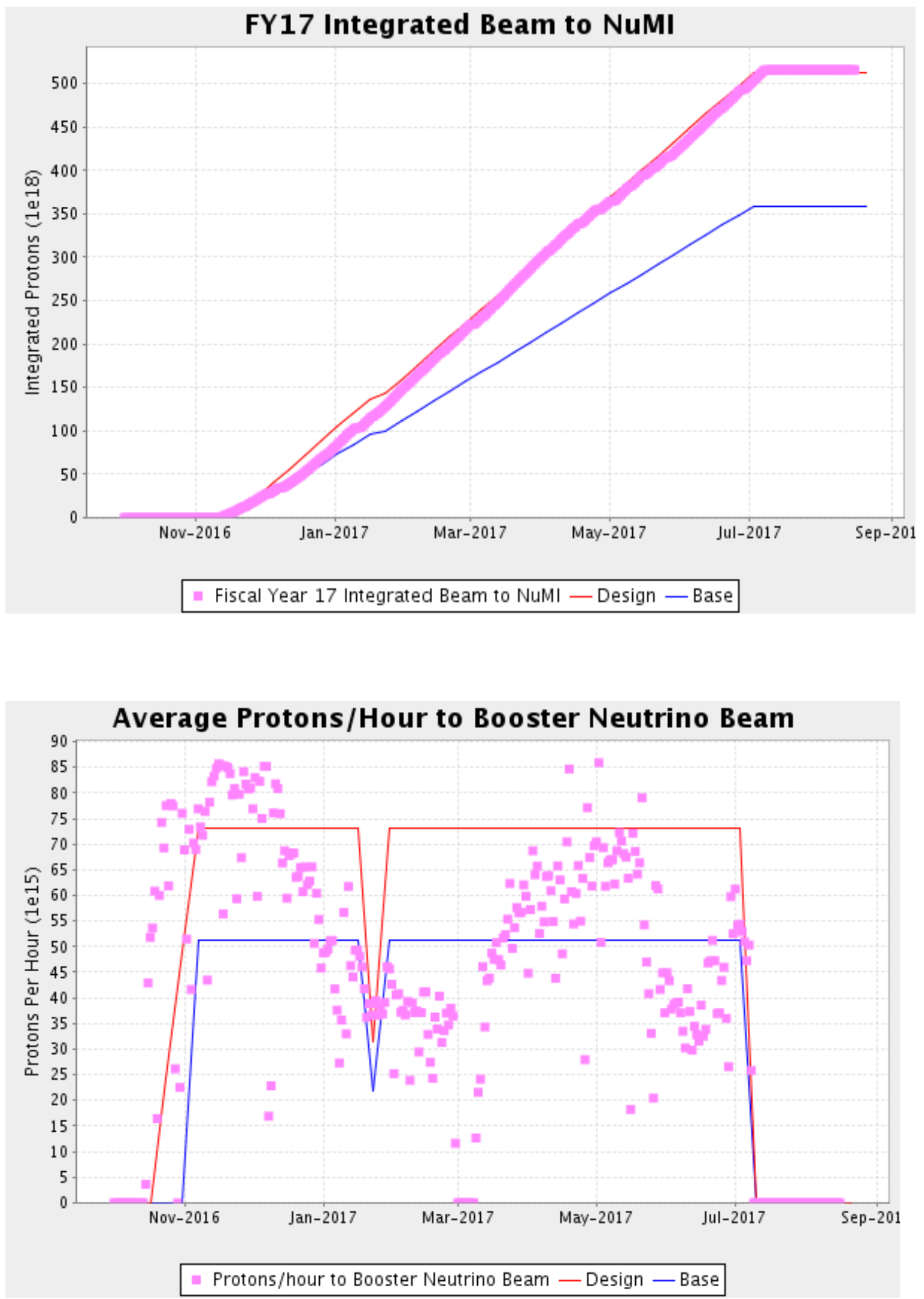


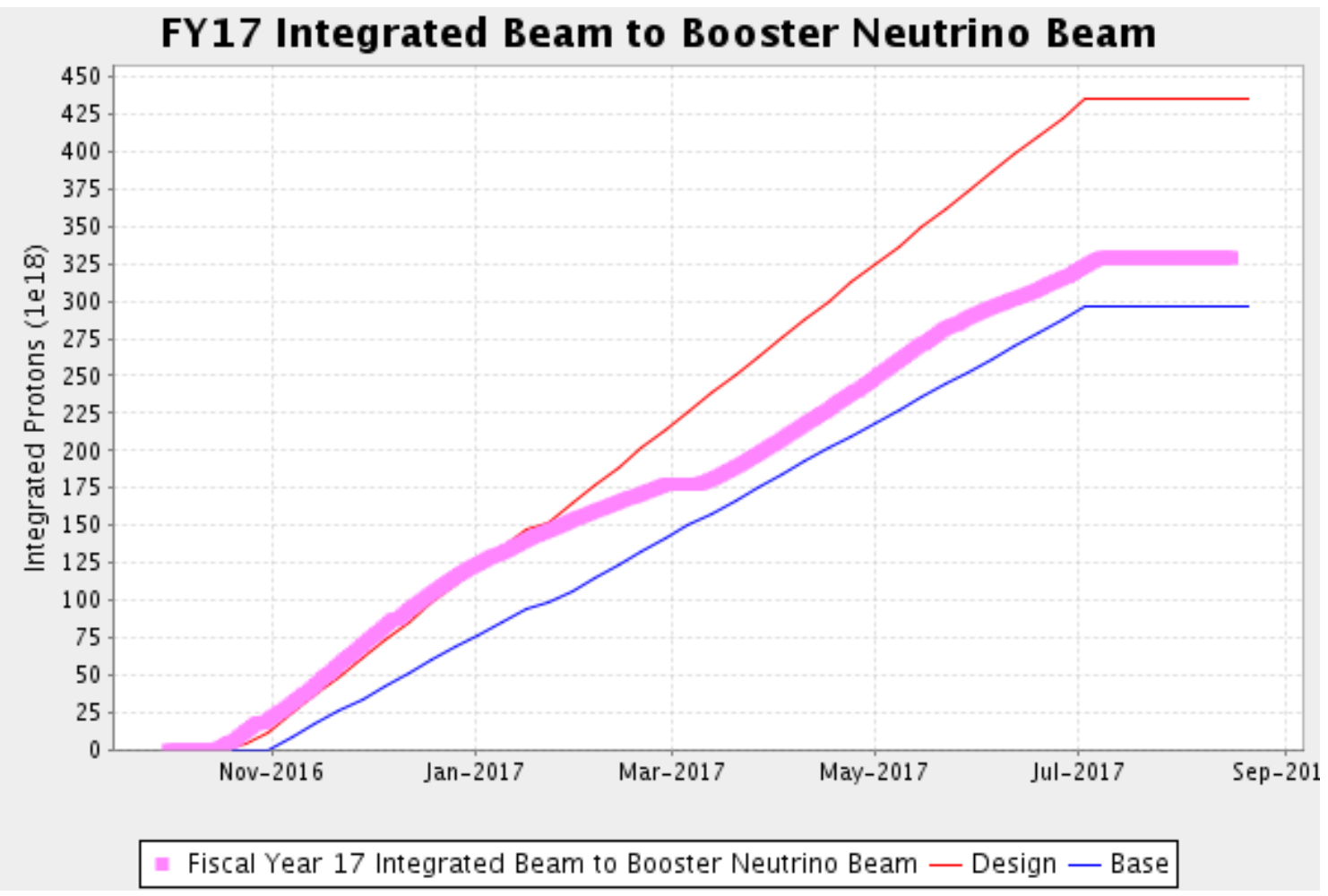

NOvA Report (M.D. Messier and P. Shanahan)

NOvA completed its third year of operations in FY 2017, published papers finalizing its 2016 muon neutrino disappearance and electron neutrino appearance results, and submitted a paper on its first sterile neutrino search result. NOvA switched to antineutrino data taking in February 2017, and has to-date accumulated a NuMI beam exposure in its Far Detector of $8.9 \times 10^{20}$ protons-on-target (POT) (full-detector-equivalent, accounting for beam delivered when the detector was under construction) with NuMI operating in neutrino mode, and $3.7 \times 10^{20}$ POT with NuMI operating in antineutrino mode.

NOvA research resulted in 3 Ph.D.'s being awarded in FY 2017, bringing the total to-date to 15. Six institutions - Houston, Dallas, South Alabama, Pittsburgh, California-Irvine, and Illinois Institute of Technlogy - joined NOvA in FY17, bringing the total number of collaborating institutions to 48 .

The experiment continued to staff data-taking shifts 24 hours per day without exception, with experts in data acquisition, detector controls, power supplies, and avalanche photo diode (APD) operations on-call $24 \times 7$. Shifts took place at one of 18 Remote Operations Centers (ROC) including the ROC-West at Fermilab, with new ROCs having come online in FY17 at Ash River, CalTech, SMU, University College London, and South Alabama. A team of 3 Run Coordinators serving in a rotation oversaw daily operations and coordinated activities of the shifters, experts, and support staff. The maintenance and operation of the far detector, and of the Ash River Laboratory, were performed by a crew employed by the University of Minnesota under budget and safety oversight from Fermilab, and in coordination with the collaboration operations team. 
The uptime of both detectors was generally high, with the Far Detector being live for $95.1 \%$ of protons delivered to the NuMI target during FY17, including accounting for a 12-day downtime for the Far Detector in November 2016. On November 18, a blizzard and subsequent power outage led to the overheating of the computing room at Ash River, triggering a 135 Fahrenheit sprinkler head. An uninterruptable power supply, several network switches, and 80 out of 180 nodes of the data acquisition buffer/trigger farm were lost to water damage. Once data taking resumed, the ability to capture beam neutrino data was unaffected by this loss, but the buffer depth for external supernova triggers was reduced to 17 minutes from 22 minutes. During the summer 2017 beam shutdown, the NOvA data acquisition group started to test the replacement of some of the lost nodes with former FermiGrid computing nodes that had been retired due to the expiration of their warranties.

At the Far Detector, 134 Front End Board/Thermal Electric Cooler Controller (FEB/TECC) units out of 10752 were swapped mainly due to excessive noise during FY 2017. Approximately 1000 good spares remain, and no effort has yet been made to diagnose and repair the ones that have been removed. Twenty-six avalanche photodiodes (APD) were swapped. All but about 0.06\% of the FEB/TECCs are typically active during data-taking.

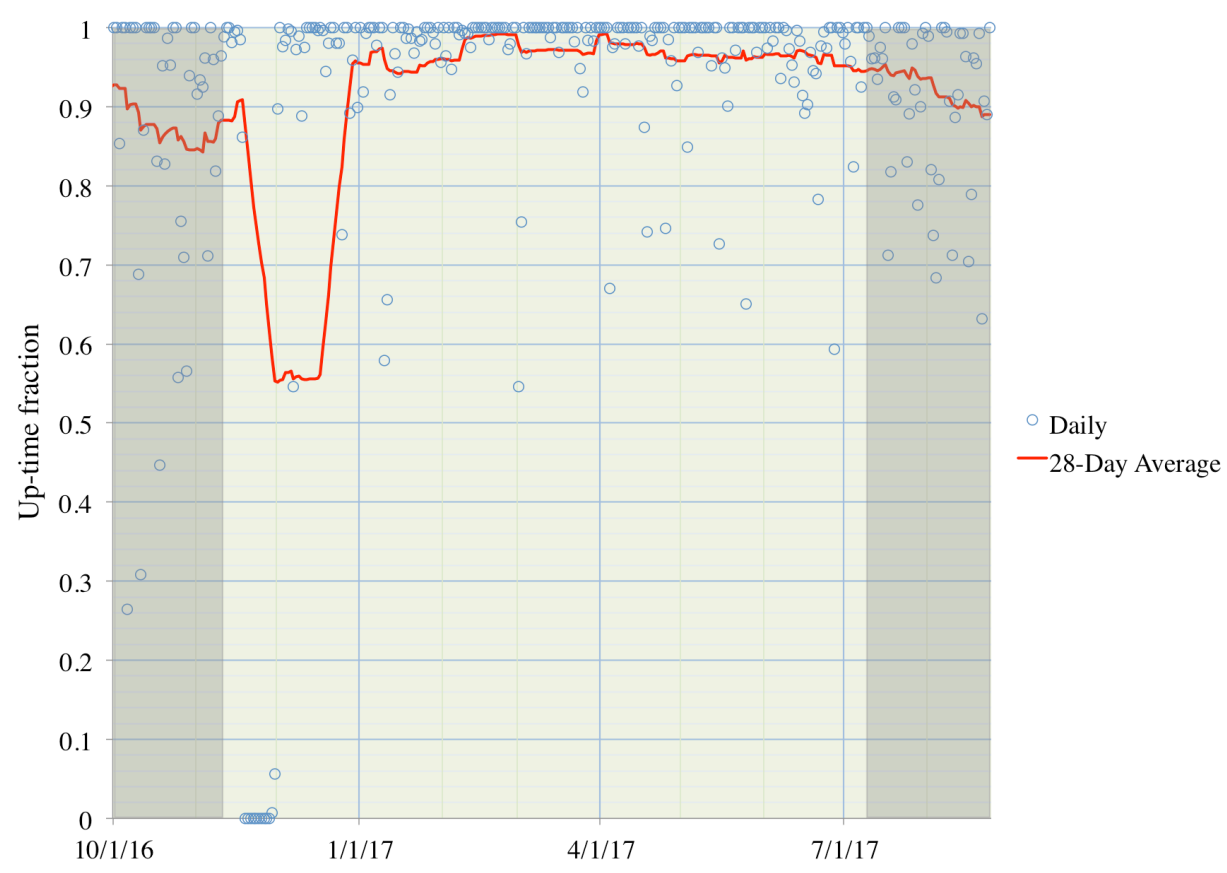

Figure N-1: The NOvA far detector uptime in FY17. This shows the daily (points) and 28-day (red line) average uptime of the NOvA detector systems including planned downtimes for maintenance during beam-off times. Accelerator shutdown periods are indicated by the shaded regions. The period of zero uptime in November corresponds to recovery from the sprinkler incident. Overall the detector operated 91\% of the time during FY17, with beam-weighted uptime of $95.1 \%$. 


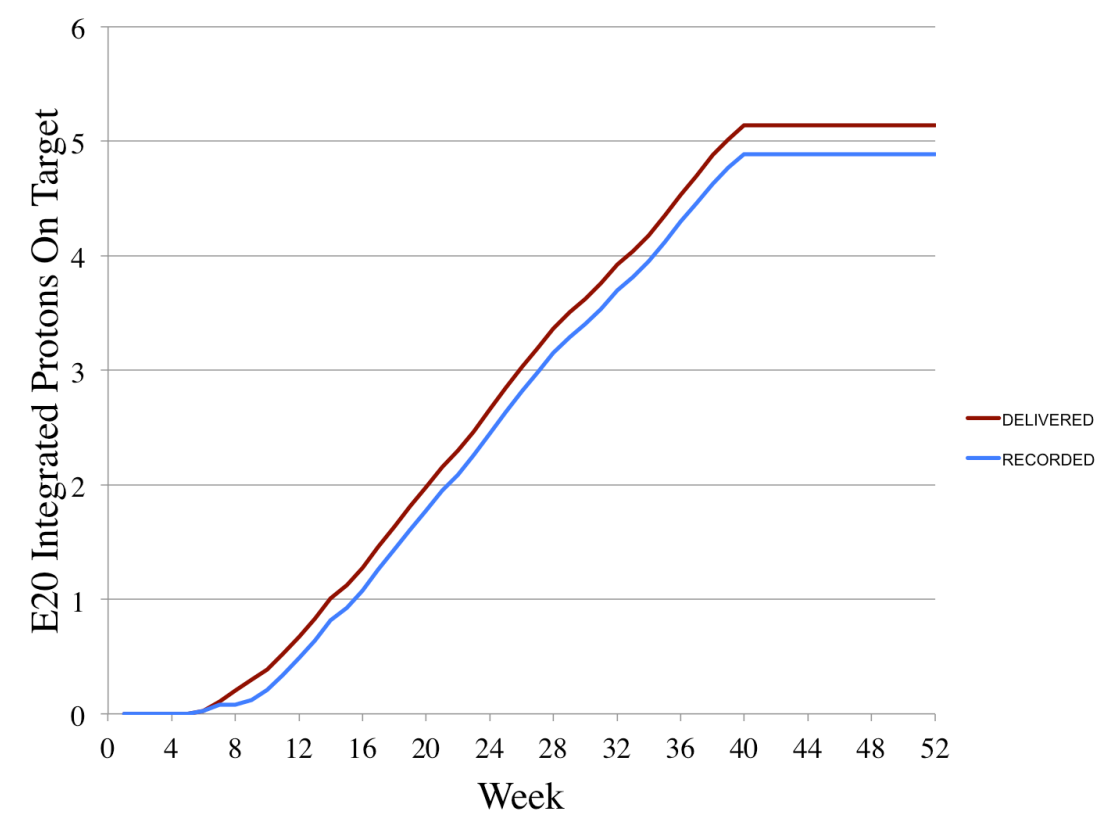

Figure N-2: The total protons on target delivered (red) and recorded (blue) by the NOvA far detector in FY17 vs week from the start of FY17. The far detector recorded data for 4.89E20 of the 5.14E20 protons delivered to the NuMI target (95.1\%). The NuMI beam was operated in anti-neutrino mode from on Feb. 20, 2017 (week 20) onward.

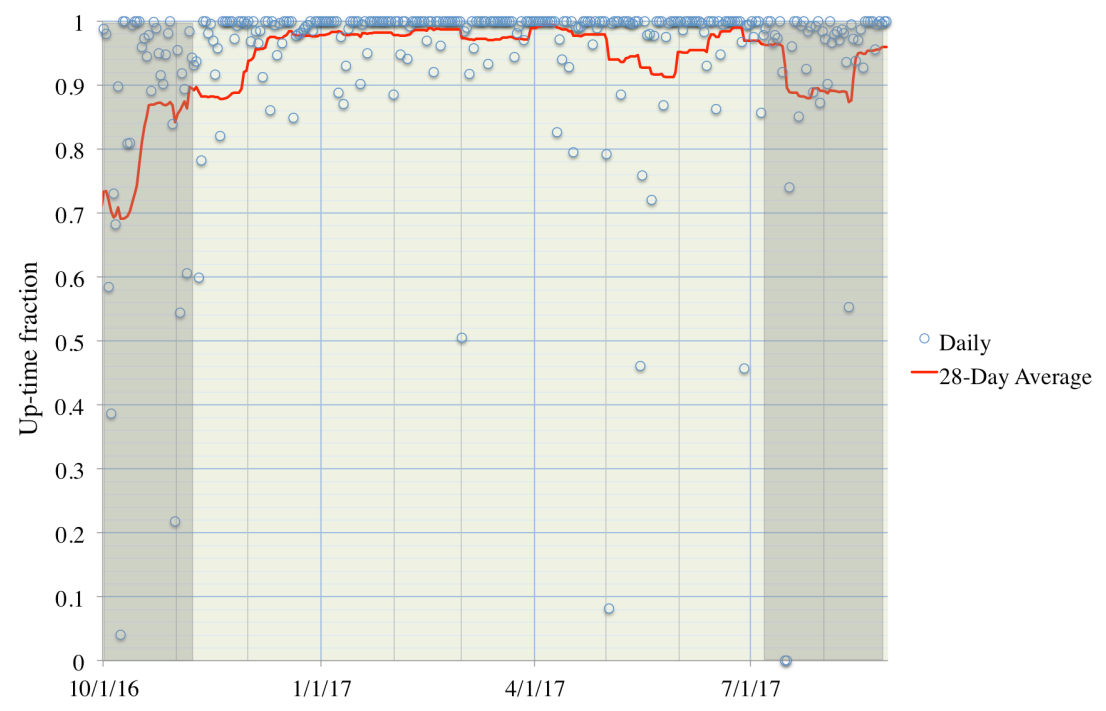

Figure N-3: The daily (points) and 28-day average (red line) NOvA near detector uptime for FY17. The shaded regions indicate beam shutdowns. Overall the detector operated $94.7 \%$ of the time during FY16, and recorded data for $99.0 \%$ of protons delivered to NuMI.

The near detector uptime was $94.7 \%$, with $99.0 \%$ beam-weighted uptime for FY 17 . The usage of spare FEBs and APDs was determined to be low enough at the end of FY16 to permit the swapping out of 29 FEBs and 88 APDs with tolerable but non-optimal noise rates, resulting in a much quieter detector in FY17. A further 25 FEBs and 5 APDs have been swapped through the end of August 2017. 
The NuMI beam - the world's most powerful neutrino beam - achieved routine operations at the design NOvA power of $700 \mathrm{~kW}$ in January 2017. In practice, $650 \mathrm{~kW}$ were typically delivered to the NuMI target when the Switch Yard 120 program was taking beam.

Figures N-1, N-2, and N-3 show the detector uptimes and delivery of protons to NuMI for FY 2016.

NOvA's second round of 3-flavor oscillation results, based on an exposure of $6.05 \times 10^{20}$ POT (full-detector equivalent) and first presented in summer 2016, were published in peer-reviewed journals. The muon neutrino disappearance result was published in Phys. Rev. Lett. 118, 151802 (2017). The 2016 electron neutrino appearance result was updated as a full joint fit of NOvA's muon neutrino disappearance and electron neutrino appearance data in Phys. Rev. Lett. 118, 231801 (2017). Both papers were selected as Editor's Suggestions, a distinction reserved for approximately $15 \%$ of Physical Review Letters.

The muon neutrino disappearance disfavors maximal mu-tau flavor mixing in the third neutrino mass state $v_{3}$, corresponding to a value of the $\theta_{23}$ mixing angle of $45^{\circ}$, at a significance of 2.6 sigma. Improvements to the muon neutrino disappearance results are under investigation for a new result that will use all $9 \times 10^{20}$ protons-on-target (full-detector equivalent) that NOvA has taken to date in neutrino mode. Potential improvements include more efficient signal selection, and using a modified procedure for applying observations in the Near Detector to the prediction of neutrino spectra in the Far Detector.
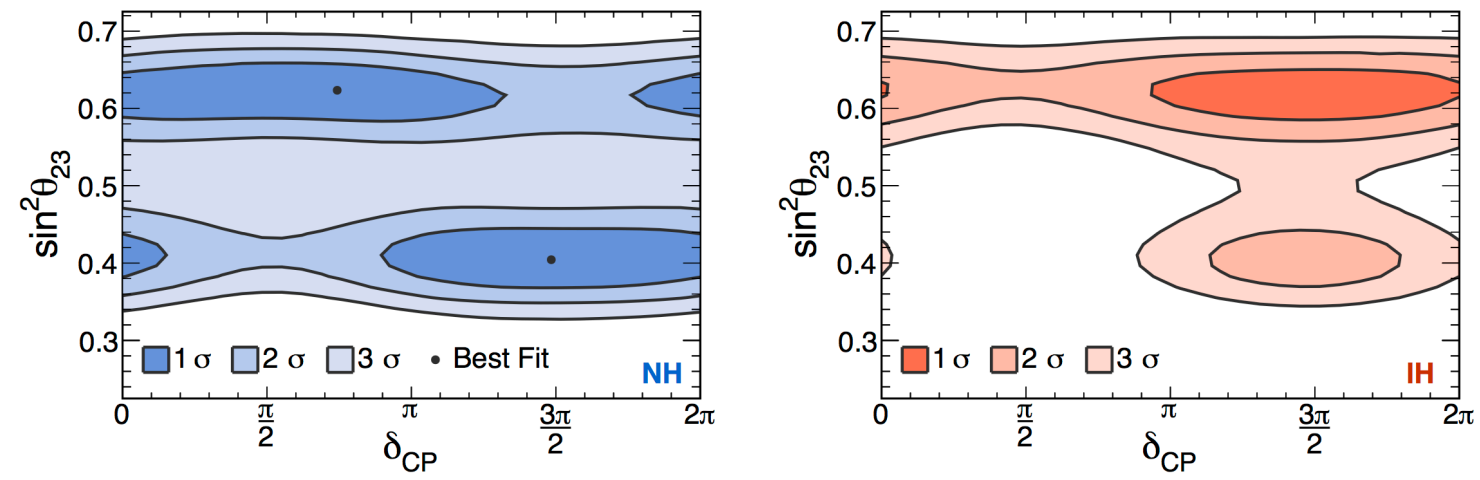

Figure N-4: The significance contours in $\sin ^{2}\left(\theta_{23}\right), \delta_{\mathrm{CP}}$, and the Mass Hierarchy (Left: Normal Hierarchy, Right: Inverted Hierarchy) for the combined NOvA muon neutrino disappearance and electron neutrino appearance fit using $\theta_{13}$ from reactor neutrino disappearance. Unshaded regions are excluded at more than 3 sigma.

The combined muon neutrino disappearance and electron neutrino appearance paper includes the results a full joint fit of the combined datasets and other improvements in extracting oscillation parameter constraints from the data, using the constraint of the $\theta_{13}$ mixing angle from measurements of electron neutrino disappearance in short-baseline reactor experiments. The NOvA result disfavors at $93 \%$ confidence level the combination of an inverted neutrino mass ordering (i.e., the case in which the least $v_{\mathrm{e}}$-like neutrino, $v_{3}$, is the lightest) and a lower octant of the mixing angle $\theta_{23}$ (i.e., more $v_{\tau}$ content than $v_{\mu}$ in $v_{3}$ ), for any value of the charge-parity 
violating phase $\delta_{\mathrm{CP}}$. Furthermore, as shown in Fig. N-4, a large region of the $\theta_{23}-\delta_{\mathrm{CP}}$ parameter space is disfavored at more than 3 sigma for an inverted neutrino mass hierarchy.

The first NOvA search for the effect of sterile neutrinos in oscillation probabilities announced in summer 2016 has been submitted for publication in Physical Review D (Rapid Communication), with preprint arXiv:1706.04592.

The NOvA collaboration has presented the effect of several potential improvements in analysis and beam operations that would signfificantly advance the physics reach of the experiment. The proposed PIP-1+ (Proton Improvement Plan) would enable up to $900 \mathrm{~kW}$ beam power to be delived to NOvA by FY2021. A reoptimization of the NuMI target that is being studied may potentially increase the neutrino flux per proton by $17 \%$. Finally, plausible improvements to the analysis over those used to produce the most recent results are assumed to yield an effective

increase in exposure of $25 \%$. In the physics scenario of Normal Mass Hierarchy, $\sin ^{2}\left(\theta_{23}\right)=0.4$, and $\delta_{\mathrm{CP}}$ near $3 \pi / 2$, which is close to the values currently favored by world data, these effects combined may make the difference in whether NOvA would reach 3 sigma mass hierarchy sensitivity before other experiments such as JUNO. In the same scenario, they would also enable NOvA to reach 3 sigma sensitivity to violation of charge-parity (CP) symmetry in neutrinos in FY24, in competition with the projections of the T2K experiment in Japan assuming increased beam power and other improvements.

E-938 / MINERvA Report (L. Fields, D. Harris)

The MINERvA experiment took physics quality data in in the NuMI Low Energy beam from November 2009 until summer 2013. In FY 2014 through FY 2016, the MINERvA experiment took data in the NuMI Medium Energy beam, and continued this data taking in FY 2017.

\section{FY 2017 Operations}

The data taken in FY 2017 was partly Medium Energy neutrino data (6 GeV peak neutrino energy) with roughly three months of antineutrino data taken just before the Summer 2017 shutdown. Between October 1, 2016 and July 7, 2017, the experiment received $1.90 \times 10^{20}$ POT in neutrino mode, and $3.16 \times 10^{20}$ POT in antineutrino mode. Since 2013 the experiment has received $16.2 \times 10^{20}$ POT in medium energy running, taken with a MINERvA Detector integrated livetime of $97.0 \%$.

During the FY 2017 running period, the collaboration continued to calibrate the detector, monitor the detector light levels, and check the reconstruction performance of both the MINERvA and MINOS detectors continuously. The experiment operates $40 \%$ of its shifts remotely, and in FY 2017 continued its check-list based shifts with no significant increase in detector downtime, thanks to new automated texting capabilities in case of DAQ outages. Figure MV-1 shows the FY 2017 protons delivered and recorded versus time, including the POT recorded when the MINERvA detector was live and, noting that some MINERvA analyses use the MINOS near detector, the POT recorded when both MINERvA and the MINOS Near Detector were live. In FY 2017, MINERvA took data with helium in the cryostat and water in the water target. There were significant problems with the MINOS magnet power supply, which meant that although the MINOS data acquisition system was working the entire detector is not considered live. That lower effective livetime is reflected in the numbers in Figure MV-1. Also, 
at the end of the neutrino run in FY16 the MINOS far detector was turned off. At this point the MINERvA collaboration took responsibility for the MINOS Near Detector, including data processing, data quality monitoring, and routine swaps of MINOS electronics boards in case of failures.

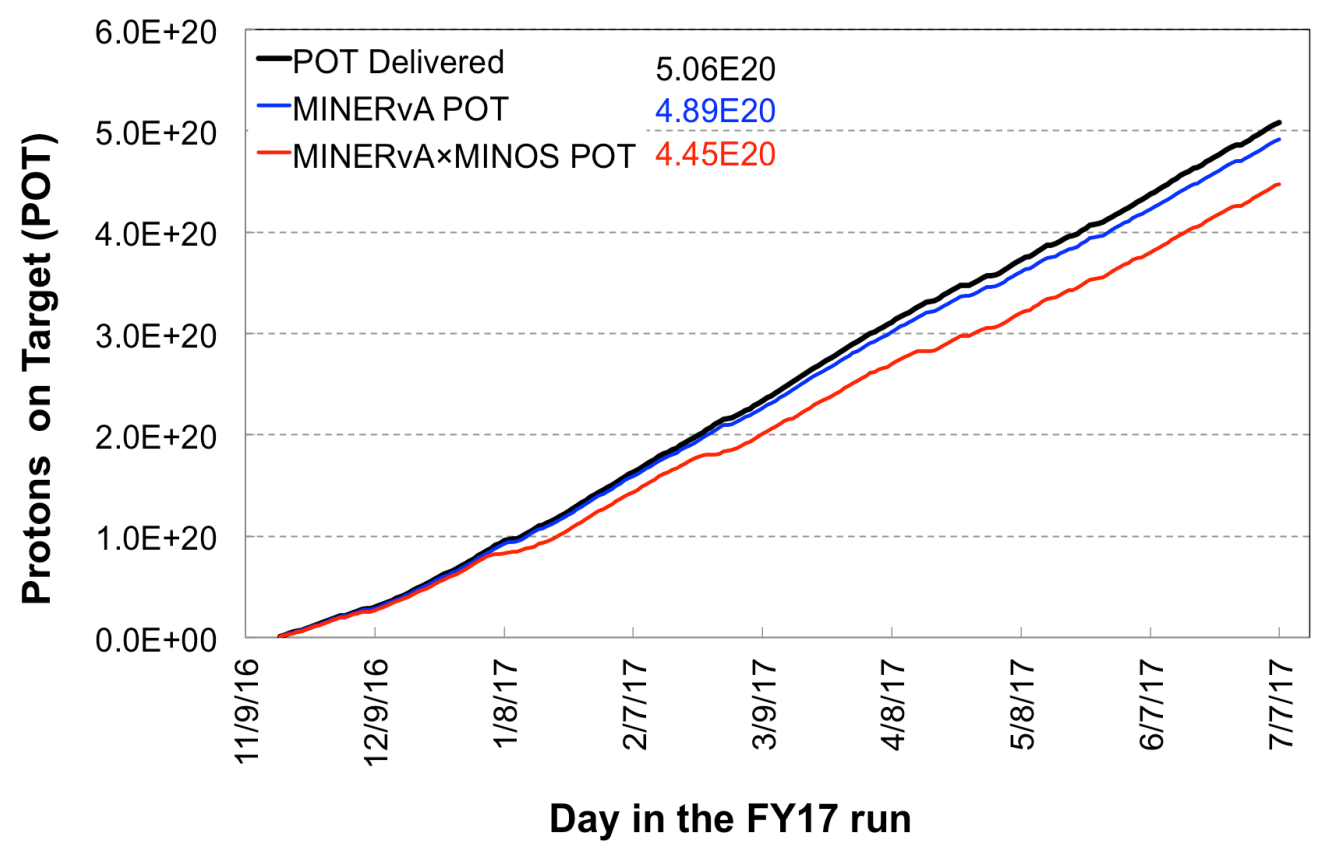

Figure MV-1: Shows the protons on target versus time, as well as the integrated livetimes of the MINERvA detector alone and the MINERvA and MINOS Near detector combined for FY17.

\section{MINERvA Construction and Installation Activities in FY 2017}

During the FY16 shutdown new data acquisition firmware was installed on the MINERvA detector. This firmware reads out lowest gain channels, which are rarely used, only once every readout cycle, which results in lower electronics deadtime during the spill. This newest firmware version has been installed since the start of the FY17 run and is operating successfully.

\section{MINERvA Results in FY 2016}

The low-energy neutrino data, which is still being analyzed, was taken to provide exclusive cross-section measurements on a variety of nuclei. Low energy events tend to have few final state particles, which allows the identification of single particles in the MINERvA detector and the measurement of exclusive channels important for current and future oscillation experiments. The MINERvA detector has a granularity that is about a factor of 10 more than the NOvA detector, and can be used to identify processes that will contribute backgrounds and signal processes in NOvA, DUNE, T2K and the SBN experiments.

By the start of 2017, the MINERvA Collaboration had published a total of 16 papers covering a broad range of neutrino and antineutrino interaction channels. By FY16 the channels included different ways of analyzing the charged current quasi-elastic interaction, a measurement of the 
inclusive charged current cross section ratios lead/scintillator, iron/scintillator, and carbon/scintillator, neutrino and antineutrino production of charged and neutral pions, coherent production of charged pions by both neutrinos and antineutrinos, and a first electron neutrino charged current quasi-elastic cross section measurement. The FY16 publications include measurements of both charged current inclusive and charged current coherent kaon production, diffractive neutral pion production, and deep inelastic scattering cross section ratios on carbon, iron and lead to scintillator.

In FY17 MINERvA published an additional 5 results, all from our low energy data set, with a sixth analysis submitted for publication at the time of this writing. These results include neutrino and antineutrino total charged current cross section measurements [Phys. Rev. D 94, 112007 (2016)], and an optimized measurement of the ratio of the antineutrino to neutrino charged current cross section [Phys. Rev. D 95, 072009 (2017)] which is of particular interest to atmospheric neutrino experiments trying to determine the mass hierarchy -- PINGU, for example). A prediction for the neutrino and antineutrino fluxes produced in the NuMI beamline was also published by the collaboration [Phys. Rev. D 94, 092005 (2016)]. This analysis takes world data on hadron production and interaction and uses that to tune a Geant-based simulation of the NuMI beamline. This prediction is being incorporated by the NOvA experiment as well.

MINERvA has also made significant progress with analysis and simulation of the medium energy neutrino data set. In particular, the simulation of accidental (beam-related) activity takes on a new importance given the very high fluxes and high proton power on the NuMI target. This activity is time-dependent even within the 10 microsecond spill because of the way that the accelerator increased its normal proton power running from $350 \mathrm{~kW}$ to $700 \mathrm{~kW}$. Simulating this meant a complete overhaul in the way the experiment overlays accidental activity from data on simulated neutrino interactions.

One FY 2017 result that has an impact on experiments outside neutrino physics is our measurement of the neutral current kaon production cross section, and in particular the cross section for events that would have a low visible energy other than the produced kaon. Atmospheric neutrino neutral current production of kaons is an intrinsic background in proton decay searches. This is because if the signal is a proton decay to a kaon and nothing else the signatures are identical if the detector only can see the kaon decay products, for example a water Cerenkov detector, or even for a fully active detector, if very little else is produced with the neutrino interaction. This process has never been measured before and the result is a data constraint on the predictions for this proton decay background. MINERvA's measurement shows that the simulation (NEUT) currently used by the Super-Kamiokande experiment underestimates the size of the background, as shown in Figure MV-2 [Phys. Rev. Lett 199, 011802 (2017)].

MINERvA has also produced measurements that constrain neutrino interaction models of the effect of the nucleus on charged current quasi-elastic interactions [arXiv:1705:03791, accepted for publication by Phys. Rev. Lett]. This process dominates the signal event rates in the NOvA and $\mathrm{T} 2 \mathrm{~K}$ experiments, and the effect of the nucleus on this process is critical because if it is not simulated correctly, the translation from the measured neutrino energy to the true neutrino energy will be biased, and therefore affect the extraction of the oscillation parameters. The events in this analysis were selected by requiring a proton and a muon to be visible in the interaction, but the muon is not required to travel to the MINOS detector. This means that a wider range of muon angles is accepted compared to MINERvA's first measurements of the 
quasi-elastic interaction. Then the proton kinematics are used to determine the momentum transferred to the nucleus $\left(\mathrm{Q}^{2}\right)$. The cross section as a function of $\left(\mathrm{Q}^{2}\right)$ for iron and lead is shown in Figure MV-3, the cross sections for carbon and hydrocarbon (scintillator) are also provided in the publication.
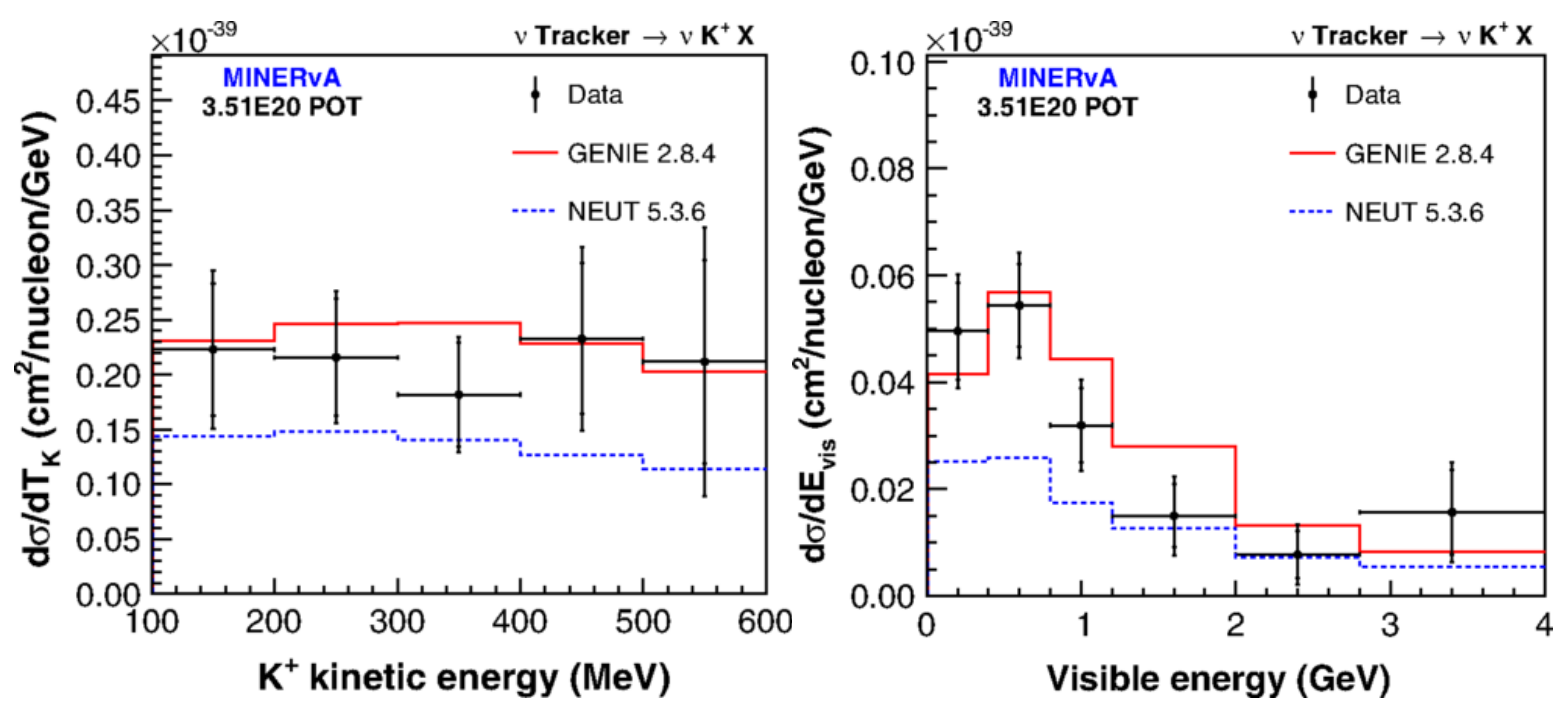

Figure MV-2: Differential neutral current kaon production cross sections as a function of both kaon kinetic energy (left) and the visible energy in the detector (right) as well as the predictions for two different event generators used by proton decay experiments: GENIE is used by DUNE, while NEUT is used by Super-Kamiokande.
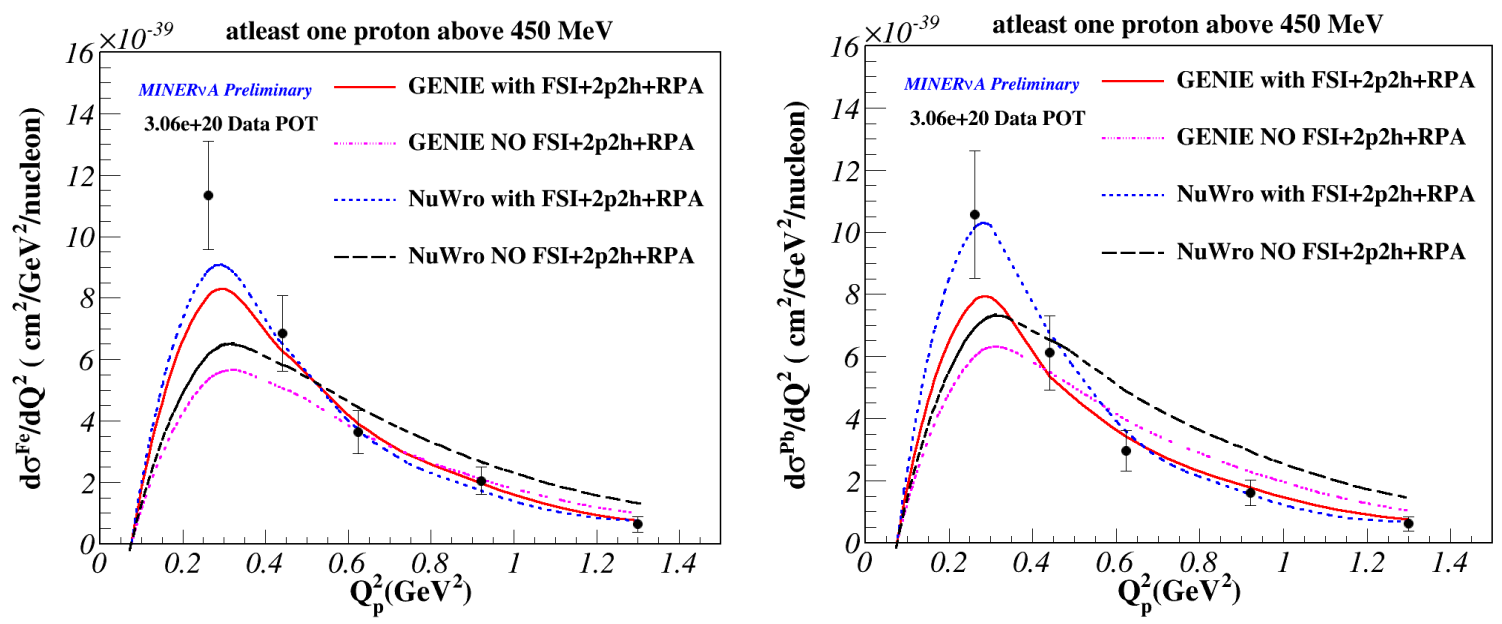

Figure MV-3: Differential quasi-elastic-like cross sections for iron (left) and lead (right) as a function of the momentum transferred to the nucleus, as measured by the proton kinematics. The measurements are shown along with several different model predictions. The comparisons show that the model used by event generator NuWro does a better job predicting the nuclear dependence of the cross section than GENIE. These models include 2particle 2-hole $(2 \mathrm{p} 2 \mathrm{~h})$ interactions which are in the signal sample yet not in either standard event generator, as well as the weak nuclear screening predicted by the Random Phase Approximation (RPA). See arXiv:1705:03791 for more details. 
Another observable for these events is the angle between the two planes defined by the neutrino and muon vectors, and the neutrino and proton vectors. For a neutrino interaction on a neutron at rest, and for no interactions of the final state proton with the nucleus, the angle between those two planes would be 180 degrees. The MINERvA detector has a few degrees resolution, which is sensitive enough to see how the presence of the nucleus would broaden this angular distribution even further. Figure MV-4 shows this angle for carbon, iron and lead, as well as the prediction coming from the models in the GENIE event generator. Although GENIE does predict this distribution correctly for carbon, it does not do as well for iron or lead.
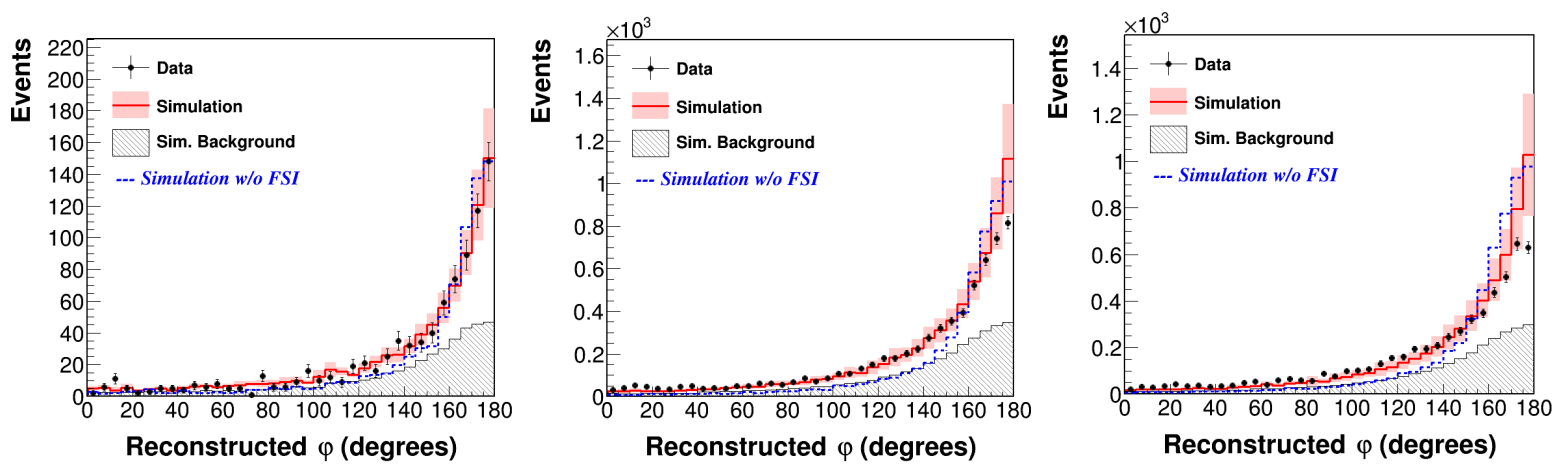

Figure MV-3: Quasi-elastic-like event rates as a function of the between the neutrino-muon and the neutrino-proton planes for carbon (left), iron (middle) and lead (right), along with the predictions for the signal and the tuned background. See arXiv:1705:03791 for more details.

Fixed Target Switchyard 120 GeV (SY120) and MTest Reports (D. Geesaman, T. Kobilarcik, P. E. Reimer, M. Rominsky, J.J. Schmidt)

The general operation of SY120 was relatively smooth. The beam for SeaQuest was operated with the same intensity as last year, as requested by the experiment, and with the maximum achievable duty factor, and manageable beam losses. The MTest beamline was operated for the FTBF program, as requested by the test beam experiments. The MCenter Beamline with a tertiary beam capability was operated as needed for the LArIAT experiment. Instrumentation improvements continued to be developed with the main emphasis on improving the BPM system. During the FY 2017 shutdown work continued on cable remediation.

\section{E906/SeaQuest Drell-Yan (P. E. Reimer)}

The SeaQuest experiment (E906) is measuring the ratio of the anti-down to anti-up quarks in the proton and the nuclear dependence of the antiquark distributions in the $\mathrm{x}_{\mathrm{Bj}}$ range of 0.1-0.5 by measuring the relative rates of proton-induced di-muon production (Drell-Yan production) on targets of ${ }^{1} \mathrm{H},{ }^{2} \mathrm{H}, \mathrm{C}, \mathrm{Fe}$ and W. Additional measurements from the same data set will include the energy loss of fast quarks in cold nuclear matter, decay angular distributions, the nuclear dependence of $\mathrm{J} /$ Psi production and a search for dark photons.

SeaQuest has been accumulating production data since the spring of 2014. At the start of the summer shutdown this year (2017) the unpolarized SeaQuest experiment had recorded at total of $1.41 \times 10^{18}$ protons on target. This is out of $3.80 \times 10^{18}$ protons delivered to the experiment. The factor of 2.7 between these numbers was caused by the variation in the instantaneous duty factor 
of the beam. RF buckets containing significantly more protons than the average (factors of 5-50) greatly increase the rate of random triggers and increase the complexity of event reconstruction, leading to a decrease in the reconstruction efficiency. To deal with these issues, the experiment uses a beam Cerenkov counter to veto events for a period of $190 \mathrm{~ns}$ around beam buckets with greater than 5-10 times the normal intensity. In the last year, AD was able to achieve the best duty factor so far, which was typically between 45 and 55\%.

During the summer shutdown in FY 2016, the experiment updated its data acquisition to significantly reduce the readout dead time by only transferring data between protons spills. The extra bandwidth allowed by this upgrade allowed the experiment to include additional trigger topographies, and thereby significantly increasing the acceptance for Drell-Yan events. The downside to this was that most of November 2016 was devoted to making this system operational. SeaQuest also lost substantial integrated luminosity between December 2016 and mid-January 2017 when the switchyard was turned off because of problems with the extraction septa and uncertainty about their repair.

SeaQuest currently has nine graduate students. Seven students have completed their $\mathrm{Ph}$. D's on the experiment. Four postdocs have active roles in the experiment and eight undergraduates are involved this year.

The collaboration is planning on switching to polarized deuterium and hydrogen targets during spring, 2018. This plan is contingent on funding and on the designs of the beam line and shielding.

Figure SQ-1: The integrated number of protons delivered to SeaQuest is show in the top (black) line as a function of time since 2014. Of those protons the center (red) line indicates those protons delivered with acceptable instantaneous duty factor. Finally the lower (blue) line shows the effect of experimental dead time on integrated protons. Note that in the final year, the lower two curves are essentially parallel due to the DAQ upgrade.

\section{The Fermilab Test Beam Facility (M. Rominsky, J.J. Schmidt)}

The Fermilab Test Beam Facility (FTBF) provides users from around the world an opportunity to test detectors in a variety of charged particle beams. The facility offers two beamlines (MTest and MCenter) to accomplish this goal. A plan view of the facility is shown in Fig. TB-1. The facility offers instrumentation to understand the beamline and infrastructure such as gas lines, high voltage lines, and signal cables. In FY 2017, results from test beam experiments were shown in 3 talks or posters at conferences. There are 3 publications and 6 articles in preparation. 


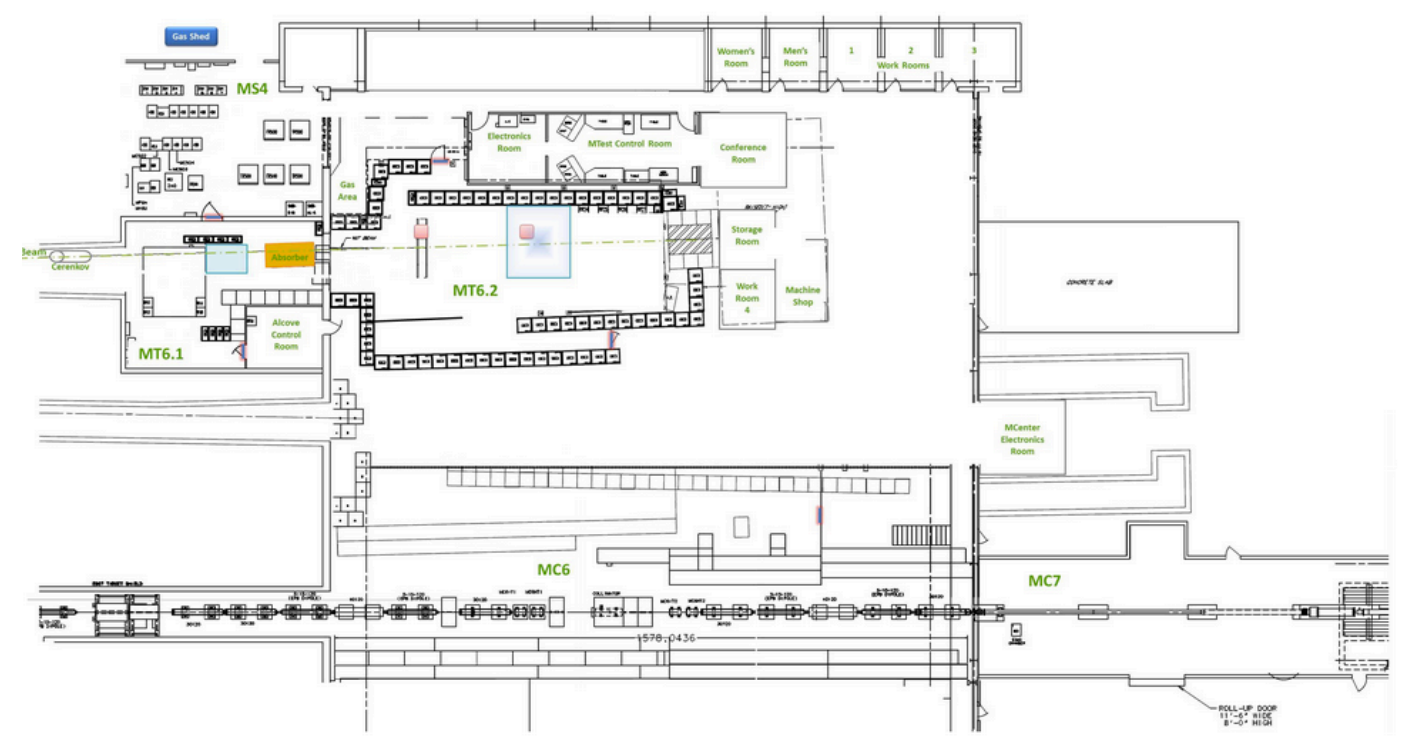

Figure TB-1: View of the Fermilab Test Beam Facility. The beamline at the top of the picture is MTest and the beamline at the bottom of the picture is MCenter. .

\section{Research Performed at the FTBF in FY 2017}

Each test beam experiment is required to prepare a Technical Scope of Work (TSW) with the laboratory in which the beam, infrastructure, and safety requirements are spelled out in detail. Five new experiments were approved in FY 2017 and started taking data. Eleven experiments returned to continue their programs. These 16 experiments are listed in Table TB- 1 and represent 141 collaborators from 49 institutions in 8 countries. Figures TB-2 and TB-3 show how the experiments broke down by user classification and research focus. We supported groups from both ATLAS and CMS, as well as groups from Brookhaven and the Relativistic Heavy Ion Collider (RHIC). The Mu2e experiment tested a variety of their detectors and are now continuing to use the facility to develop their data acquisition system. The g-2 experiment used the facility to conduct rate of rise tests for their straw-tube detectors. We supported the neutrino experiments LArIAT and LAToF for ProtoDUNE. We also had several groups come in to test general detector research and development. Full information from these groups will be included in the annual test beam report (in preparation).

The experiments fully utilized the MTest beamline for data taking purposes out of the 25 weeks that were available with beam during FY17. There was an unexpected 5 week downtime that hampered user time. However, we were able to reschedule all users. Counting each user per week yields 55 weeks of experiment time during FY 2016, with multiple users in the beam line at the same time. The MCenter beamline was used for data taking for the LArIAT experiment (T1034) and for the Large Area Time of Flight for ProtoDUNE (T1075). LArIAT ran well and is proposing a short run for the Fall of FY18 to test pixel detectors. 


\begin{tabular}{|c|c|}
\hline $\begin{array}{l}\text { Experiment } \\
\text { Number }\end{array}$ & Description \\
\hline T1041 & CMS Forward Calorimetry R\&D \\
\hline T0992 & Radiation Hard Sensors for the HL-LHC \\
\hline T1209 & CMS Outer Tracker R\&D \\
\hline T1065 & Secondary Emitters Study \\
\hline T1068 & Beam Tests of the SVX4 Telescope \\
\hline T1043 & Mu2e Cosmic Ray Veto Detector \\
\hline T1044 & sPHENIX Calorimetry Tests \\
\hline T1224 & ATLAS Pixel Telescope Tests \\
\hline T1274 & LAFTBFToF For ProtoDUNE \\
\hline T1273 & LHCb VELO High Rate Test Beam \\
\hline T1042 & g-2 Straw Tracker Vacuum Tests \\
\hline T1315 & $\begin{array}{l}\text { LBNF Spectrometer: Prelim RF Bucket } \\
\text { Occupancy Measurement }\end{array}$ \\
\hline T1314 & Muon Strips for Future Colliders \\
\hline T1272 & EM Mini Calorimeter \\
\hline T267 & IceCube DOM Beam Test \\
\hline T1034 & LArIAT: Liquid Argon in a Test Beam \\
\hline
\end{tabular}

Table TB-1: Test Beam experiments performed in FY 2017. 


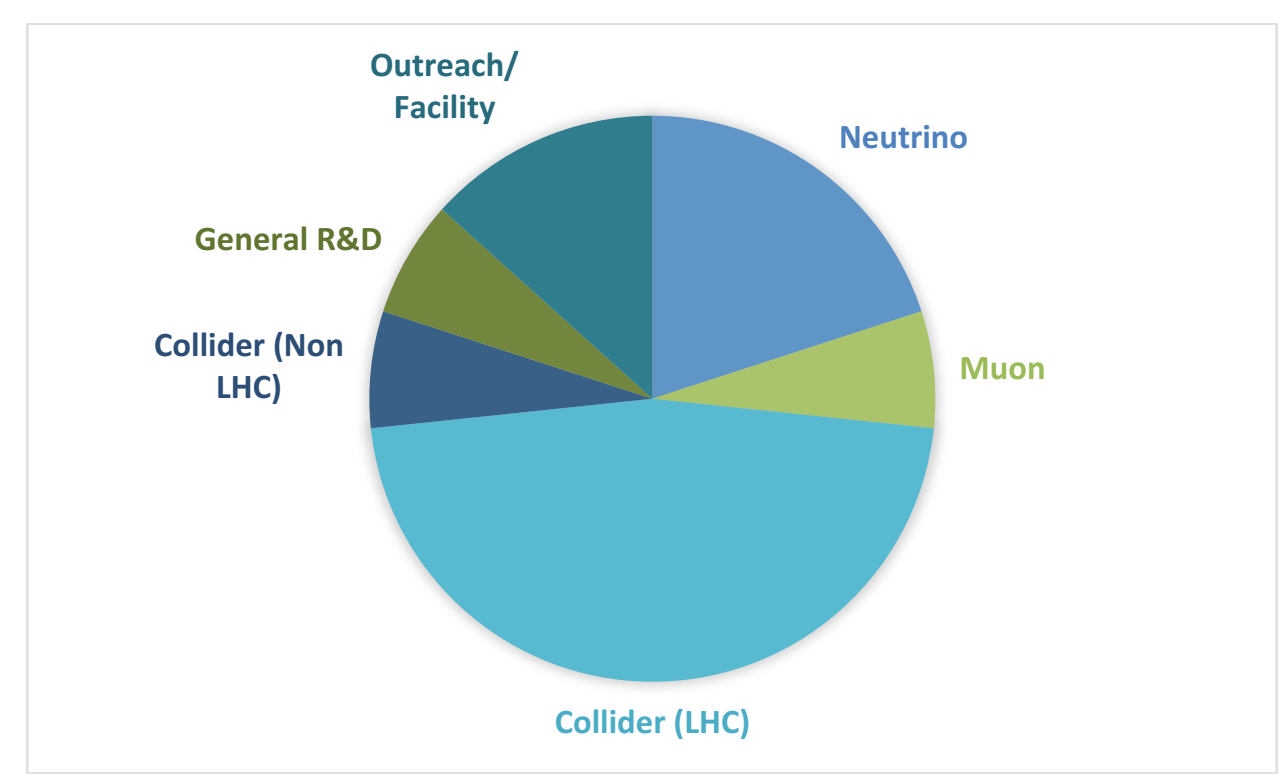

Figure TB-2: The research focus of the different groups that came to the test beam in FY2017. We support a broad range of users.

MicroBooNE report (B.T. Fleming and G.P. Zeller)

MicroBooNE completed its second year of operations in FY 2017. As of the start of the accelerator shutdown in summer 2017, MicroBooNE has collected a total of $6.5 \times 10^{20}$ POT $\left(6.2 \times 10^{20}\right.$ POT recorded) of Booster Neutrino beam (BNB) with the detector on (Figure M-1). Combined detector and DAQ uptime for the year has been $>95 \%$. In FY17, MicroBooNE operations were handled by series of 5 Run Coordinators from Yale, Harvard, IIT, PNNL, and Syracuse University. Shifts were performed across several shift-taking sites including ROC-W at Fermilab and 10 remote shift centers in the U.S., U.K, and Switzerland. This year, MicroBooNE benefitted from the installation of new interlock gates in the Fermilab Main Injector that, for the first time in Fermilab history, allows the Booster Neutrino beamline to run when part of NuMI is down for repair. MicroBooNE collected an extra $0.5 \times 10^{20}$ POT in this mode. Also this past year, a spare BNB horn was constructed and test pulsed. The associated target and instrumentation is near completion. 


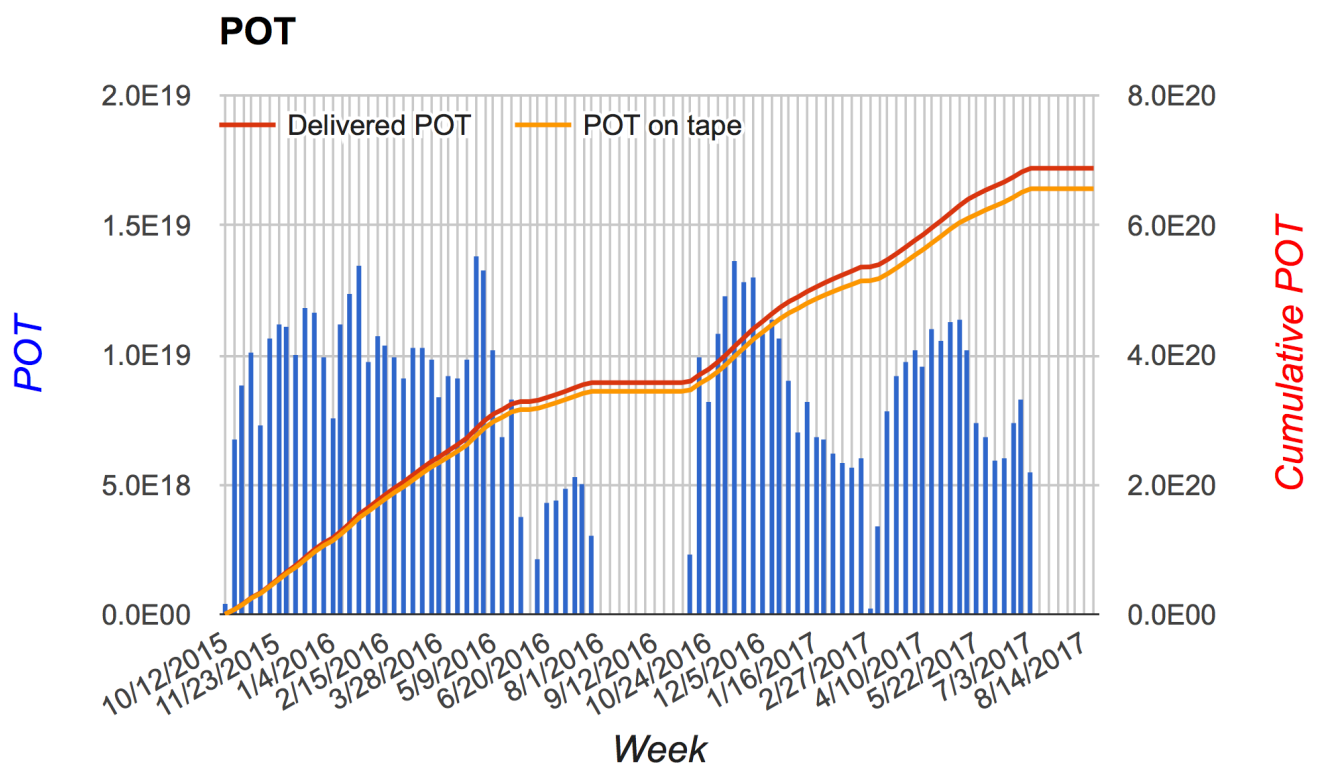

Figure M-1: Total protons on target delivered (red) and recorded (orange) by MicroBooNE since the start of operations in October 2015. Note that the MicroBooNE detector was not running during all of these periods (see note in text about such periods in February and May 2017).

MicroBooNE detector operations have been very stable throughout the year with the exception of a 28 day period in late January/early February and a 14 day period in May. The collaboration spent the month of February investigating a sudden onset of drift high voltage problems that were eventually traced to a faulty connection that had developed between the drift high voltage feedthrough and the cathode plane in the TPC. After carefully repositioning the drift high voltage feedthrough, the detector has been since operating stably at our nominal operating drift voltage of $70 \mathrm{kV}$. The collaboration learned a lot in this trouble-shooting process and shared lessons learned with other liquid argon TPC experiments in a joint SBN/DUNE workshop in May 2017. At this joint "lessons learned" workshop, MicroBooNE delivered a series of presentations ranging in topics from drift high voltage feedthrough connectivity to data analysis techniques. Also for a 14 day period in May, some new issues developed with liquid argon recirculation in the MicroBooNE cryogenics system following a power outage - stable operations have since resumed, but the system has had to undergo substantial trouble-shooting by Fermilab Neutrino Division cryogenics engineers in concert with MicroBooNE collaboration input.

In addition to learning a lot about liquid argon TPC operations over the past year, MicroBooNE has also been busy implementing several detector upgrades. This work included the installation of a $\$ 1 \mathrm{M}$ cosmic ray muon tracking system from the University of Bern, Switzerland that surrounds the cryostat to help further mitigate cosmic ray backgrounds (Figure M-2). Residual noise sources in the detector were reduced via replacement of service boards in each of the TPC signal feedthroughs and installation of a second drift high voltage low pass filter. Other improvements include an upgrade to the beam timing signals arriving at the detector and the addition of a new heavy sterile neutrino trigger. MicroBooNE is also in the process of commissioning a new supernova neutrino readout stream, the first time such a capability has been deployed in a liquid argon TPC. 


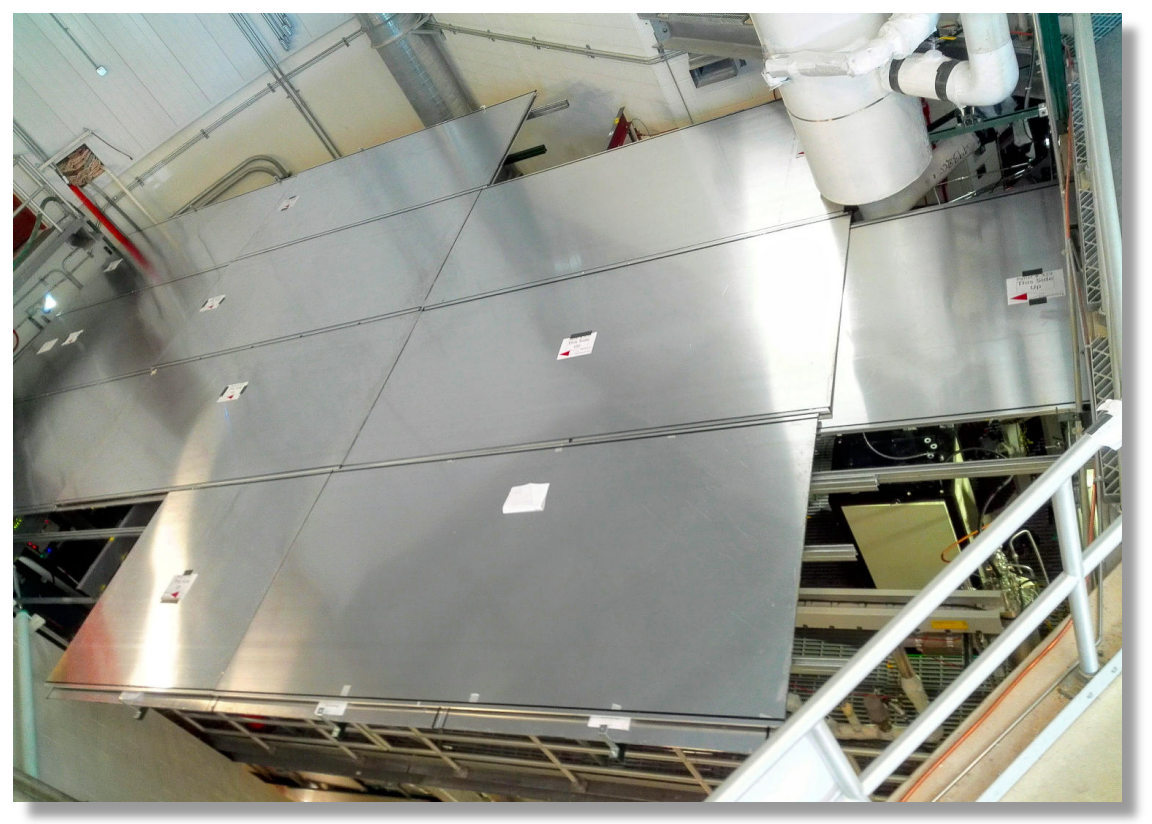

Figure M-2: Completed cosmic ray tagger system that is now surrounding the MicroBooNE detector in LArTF.

In FY17, the MicroBooNE collaboration launched our first large scale Monte Carlo production that used data as input to tuning our simulations and reconstruction. The collaboration held an annual MicroBooNE reconstruction retreat at the University of Cincinnati in March 2017 to review these updated samples and their impact on our physics analyses.

MicroBooNE had a very prolific year. The MicroBooNE collaboration submitted a total of 7 papers to journals in FY17. This included papers describing the design and construction of the MicroBooNE detector (R. Acciarri et al., JINST 12, P02017, 2017), the first application of machine learning techniques on liquid argon TPC data (R. Acciarri et al., JINST 12, P03011, 2017), the first extraction of multiple Coulomb scattering parameters for argon using cosmic ray muons (P. Abratenko et al., arXiv:1703.06187, submitted to JINST), the largest sample of Michel electrons ever reconstructed in a liquid argon TPC as a first step towards understanding MicroBooNE's performance in reconstructing electrons (R. Acciarri et al., arXiv:1704.02927, accepted by JINST), a comprehensive summary of all noise sources and their mitigation in MicroBooNE (R. Acciarri et al., JINST 12, P08003, 2017), measurement of cosmic ray muon reconstruction efficiencies (R. Acciarri et al., arXiv:1707.09908, submitted to JINST), and a detailed description of one of our main reconstruction packages called Pandora (R. Acciarri et al., arXiv:1708.03135, submitted to Eur. Phys. J. C.). MicroBooNE also presented its first Wine \& Cheese seminar at Fermilab on July 28, 2017.

\section{Acknowledgement}

This report gives a brief summary of the performance and output of the accelerator complex and associated accelerator-based experiments during FY 2017. It therefore summarizes the work of many people from Fermilab and from the collaborating institutions. The credit for the successful outcome of the FY 2017 running is shared amongst many. 